\title{
The Zeros of the Bergman Kernel for Some Reinhardt Domains
}

\author{
Jong-Do Park \\ Department of Mathematics and Research Institute for Basic Sciences, Kyung Hee University, Seoul 130-701, Republic of Korea
}

Correspondence should be addressed to Jong-Do Park; mathjdpark@khu.ac.kr

Received 26 July 2016; Accepted 31 August 2016

Academic Editor: Gestur Ólafsson

Copyright (C) 2016 Jong-Do Park. This is an open access article distributed under the Creative Commons Attribution License, which permits unrestricted use, distribution, and reproduction in any medium, provided the original work is properly cited.

We consider the Reinhardt domain $D_{n}=\left\{(\zeta, z) \in \mathbb{C} \times \mathbb{C}^{n}:|\zeta|^{2}<\left(1-\left|z_{1}\right|^{2}\right) \cdots\left(1-\left|z_{n}\right|^{2}\right)\right\}$. We express the explicit closed form of the Bergman kernel for $D_{n}$ using the exponential generating function for the Stirling number of the second kind. As an application, we show that the Bergman kernel $K_{n}$ for $D_{n}$ has zeros if and only if $n \geq 3$. The study of the zeros of $K_{n}$ is reduced to some real polynomial with coefficients which are related to Bernoulli numbers. This result is a complete characterization of the existence of zeros of the Bergman kernel for $D_{n}$ for all positive integers $n$.

\section{Introduction}

Let $D$ be a bounded domain in $\mathbb{C}^{n}$. The space $L_{a}^{2}(D)$ is denoted by the set of all holomorphic functions $f$ on $D$ satisfying

$$
\int_{D}|f(z)|^{2} d V(z)<\infty
$$

where $d V$ is the volume measure on $D$. For any $z \in D, \Phi_{z}$ : $L_{a}^{2}(D) \rightarrow \mathbb{C}$ defined by $\Phi_{z}(f)=f(z)$ is a bounded linear functional on $L_{a}^{2}(D)$. By Riesz representation theorem, there exists the unique element $K_{z}(\cdot) \in L_{a}^{2}(D)$ such that $\Phi_{z}(f)=$ $\left\langle f(\cdot), K_{z}(\cdot)\right\rangle$; namely,

$$
f(z)=\int_{D} f(w) \overline{K_{z}(w)} d V(w),
$$

for all $f \in L_{a}^{2}(D)$. Define the Bergman kernel function $K_{D}(z, w):=\overline{K_{z}(w)}$ for $D$. It is defined for arbitrary bounded domains, but it is difficult to obtain the explicit form of the Bergman kernel for general bounded domains. Recently, the Bergman kernels for various domains have been computed explicitly in [1-6].

Over the last decade the Hartogs domain

$$
\widehat{\Omega}_{m}:=\left\{(\zeta, z) \in \mathbb{C}^{m} \times \Omega:\|\zeta\|^{2}<p(z)\right\}
$$

was investigated, where $p$ is a suitably chosen continuous function on a bounded domain $\Omega$. The Bergman kernel for
$\widehat{\Omega}_{m}$ was obtained explicitly in [7] when $\Omega$ is an irreducible bounded symmetric domain. This result was generalized to the cases when $\Omega$ is the product of bounded symmetric domains in [8] and when $\Omega$ is a bounded homogeneous domain in [9].

Also the problem of determining whether the Bergman kernels are zero-free has been a well-known open problem in several complex variables ever since Lu Qi-Keng raised the question related to the existence of Bergman representative coordinates. If the Bergman kernel $K_{D}(z, w)$ for a bounded domain $D$ is zero-free for all $(z, w) \in D \times D$, then $D$ is called the Lu Qi-Keng domain. One can see many examples of Lu Qi-Keng domains and non-Lu Qi-Keng domains in [8-13].

If $\Omega$ is symmetric [8] or homogeneous [9], then the main part of the Bergman kernel for $\widehat{\Omega}_{m}$ is the polynomial whose coefficients are written as the forms containing the Stirling number of the second kind. The Routh-Hurwitz theorem (see Lemma 11) gives the condition that a real polynomial has no zeros in the closed right half-plane, and using this criterion we have the algorithmic method of determining whether the Hartogs domain $\widehat{\Omega}_{m}$ is a Lu Qi-Keng domain or not. The existence of zeros of all Hartogs domains $\widehat{\Omega}_{m}$ is classified in $[8,9]$ only when the dimension of the base domain $\Omega$ is low (less than 4). However it looks hard to study Lu Qi-Keng problem for all dimensions, since Routh-Hurwitz theorem involves too many terms when the order of the polynomial is large. 
In this paper we consider the Reinhardt domain $D_{n} \subset$ $\mathbb{C}^{n+1}$ defined by

$$
\begin{aligned}
D_{n} & :=\left\{(\zeta, z)=\left(\zeta, z_{1}, z_{2}, \ldots, z_{n}\right) \in \mathbb{C} \times \mathbb{C}^{n}:|\zeta|^{2}\right. \\
& \left.<\prod_{j=1}^{n}\left(1-\left|z_{j}\right|^{2}\right)\right\} .
\end{aligned}
$$

From Theorem 2.5 in [8], the Bergman kernel for $D_{n}$ can be obtained explicitly as the following.

Theorem 1. The Bergman kernel $K_{n}$ for $D_{n}$ is written as

$$
\begin{aligned}
& K_{n}((\zeta, z),(\eta, w)) \\
& \quad=\frac{1}{\pi^{n}} \prod_{l=1}^{n} \frac{1}{\left(1-z_{l} \bar{w}_{l}\right)^{3}} F_{n}\left(\frac{\langle\zeta, \eta\rangle}{\prod_{l=1}^{n}\left(1-z_{l} \bar{w}_{l}\right)}\right),
\end{aligned}
$$

where

$$
F_{n}(t)=\sum_{j=1}^{n}(-1)^{n-j} S(n, j) \frac{(j+1) !}{(1-t)^{j+2}},
$$

where $S(n, j)$ is the Stirling number of the second kind.

In Section 2, we prove Theorem 1 using the result in [8] and express $a(n, \ell)$ in terms of the coefficients of a certain generating function (see Theorem 8). We use the well-known formal series

$$
\frac{\left(e^{t}-1\right)^{j}}{j !}=\sum_{n=0}^{\infty} S(n, j) \frac{t^{n}}{n !}
$$

for exponential generating function, where $S(n, j)$ is the Stirling number of the second kind.

For the study of the existence of zeros of $K_{n}((\zeta, z),(\eta, w))$, we need to define $\widetilde{F}_{n}(t)$ and $G_{n}(\tau)$ by

$$
\begin{aligned}
\widetilde{F}_{n}(t) & =(1-t)^{2} F_{n}(t), \\
G_{n}(\tau) & =\widetilde{F}_{n}\left(1-\frac{1}{\tau+1 / 2}\right) \\
& =\sum_{j=1}^{n}(-1)^{n-j} S(n, j)(j+1) !\left(\tau+\frac{1}{2}\right)^{j} .
\end{aligned}
$$

The zero set of the Bergman kernel $K_{n}((\zeta, z),(\eta, w))$ with $(\zeta, z),(\eta, w) \in D_{n}$ reduces to the zero set of the polynomial $G_{n}(\tau)$ with $\operatorname{Re} \tau<0$. Now we write

$$
\begin{aligned}
G_{n}(\tau)= & \sum_{k=0}^{n} a(n, n-k) \tau^{k} \\
= & a(n, n)+a(n, n-1) \tau+\cdots+a(n, 1) \tau^{n-1} \\
& +a(n, 0) \tau^{n} .
\end{aligned}
$$

In Section 3, we introduce the Routh-Hurwitz theorem that is efficient on checking whether the real polynomial $G_{n}(\tau)$ has zeros in the right half plane. Using the generating form of coefficients of $G_{n}(\tau)$ (see Proposition 12), we will show that if $n \geq 3$, then $G_{n}(\tau)$ does not satisfy Routh-Hurwitz conditions, so we obtain the following main result of this paper.

Theorem 2. The Bergman kernel for $D_{n}$ is zero-free if and only if $n \leq 2$.

For the proof of Theorem 2, we will show that if $n \geq$ 3 , then at least one of $a(n, n), a(n, n-1)$, or $a(n, n-2)$ is negative (see Theorem 13). In Section 4, we discuss the properties of $a(n, \ell)$ and prove Theorem 13 using properties of the Bernoulli numbers and Genocchi numbers.

Remark 3. In Theorem 5.2(ii) and Theorem 5.3 of [8], one can see that $K_{2}$ has no zeros and $K_{3}$ has zeros. The main contribution, Theorem 2 in this paper, is the complete classification of the answer to the Lu Qi-Keng problem for $D_{n}$ for all dimensions $n$.

\section{Explicit Form of the Bergman Kernel for $D_{n}$}

In [8], we know the explicit form of the Bergman kernel for Cartan-Hartogs domain $\widehat{\Omega}_{m}$ in the case when

$$
\begin{aligned}
\Omega & =\Omega_{1} \times \cdots \times \Omega_{n}, \\
p\left(z_{1}, \ldots, z_{n}\right) & =\prod_{l=1}^{n} N_{\Omega_{l}}\left(z_{l}, z_{l}\right)^{\mu_{l}}, \quad \mu_{l}>0,
\end{aligned}
$$

where $N_{\Omega_{l}}$ is the generic norm with respect to the bounded symmetric domain $\Omega_{l}$. Using the numerical invariants $a, b, r$ with respect to the bounded symmetric domain, we define the Hua polynomial

$$
\chi(s)=\prod_{j=1}^{r}\left(s+1+(j-1) \frac{a}{2}\right)_{1+b+(r-j) a},
$$

where $(s)_{k}=s(s+1)(s+2) \cdots(s+k-1)$ for $k \geq 1$ and $(s)_{0}=1$. Then for any $\mu>0$, we define $c(\mu, j)$ by

$$
\frac{\chi(k \mu)}{\chi(0)}=\sum_{j=0}^{d} c(\mu, j)(k+1)_{j},
$$

where $d:=r+r b+r(r-1) a / 2$. We also define $d_{j}^{j_{1}, \ldots, j_{n}}$ satisfying

$$
\prod_{l=1}^{n}(k+1)_{j_{l}}=\sum_{j=\max \left(j_{1}, \ldots, j_{n}\right)}^{j_{1}+\cdots+j_{n}} d_{j}^{j_{1}, \ldots, j_{n}}(k+1)_{j} .
$$

Theorem 4 (see [8]). Let $\widehat{\Omega}_{m}:=\left\{(\zeta, z) \in \mathbb{C}^{m} \times \Omega:\|\zeta\|^{2}<\right.$ $p(z)\}$, where $\Omega$ and $p$ are defined as in (10). Then the Bergman kernel for $\widehat{\Omega}_{m}$ is given by

$$
\begin{aligned}
& \widehat{K}_{m}((\zeta, z),(\eta, w)) \\
& \quad=\frac{1}{m !} \prod_{l=1}^{n} \frac{K_{\Omega_{l}}\left(z_{l}, w_{l}\right)}{N_{\Omega_{l}}\left(z_{l}, w_{l}\right)^{m \mu_{l}}} F_{n}\left(\frac{\langle\zeta, z\rangle}{\prod_{l=1}^{n} N_{\Omega_{l}}\left(z_{l}, w_{l}\right)^{\mu_{l}}}\right),
\end{aligned}
$$


where

$$
\begin{gathered}
F_{n}(t)=\sum_{j_{1}=0}^{\operatorname{dim} \Omega_{1}} \cdots \sum_{j_{n}=0}^{\operatorname{dim} \Omega_{n}} \prod_{l=1}^{n} c\left(\mu_{l}, j_{l}\right) \\
\cdot \sum_{j=\max \left(j_{1}, \ldots, j_{n}\right)}^{j_{1}+\cdots+j_{n}} d_{j}^{j_{1}, \ldots, j_{n}} \frac{(j+m) !}{(1-t)^{j+m+1}} .
\end{gathered}
$$

For any $n \geq k$, the number of the partitions of $\{1,2, \ldots, n\}$ into $k$ blocks is denoted by $S(n, k)$ and called the Stirling number of the second kind.

Lemma 5 (see [14]). For any positive integer $p$, it holds that

$$
x^{p}=\sum_{j=1}^{p} S(p, j)(x-j+1)_{j} .
$$

Proof of Theorem 1. For any $1 \leq \ell \leq n$, let $\Omega_{l}=\{z \in \mathbb{C}:|z|<$ $1\}$ be the unit disk and $m=\mu_{1}=\cdots=\mu_{n}=1$. Then $\widehat{\Omega}_{m}=D_{n}$ and

$$
\begin{aligned}
r & =1, \\
a & =2, \\
b & =0, \\
g & =2, \\
N_{\Omega_{l}}(z, w) & =1-z \bar{w}, \\
K_{\Omega_{l}}(z, w) & =(1-z \bar{w})^{-2} .
\end{aligned}
$$

Since the Hua polynomial is $\chi(s)=s+1$, by (12) we have

$$
k+1=c(1,0)+c(1,1)(k+1),
$$

so that $c(\mu, 0)=0$ and $c(\mu, 1)=1$.

Thus the function $F_{n}(t)$ in Theorem 4 is reduced to

$$
F_{n}(t)=\sum_{j=1}^{n} d_{j}^{1, \ldots, 1} \frac{(j+1) !}{(1-t)^{j+2}} .
$$

Now we claim that $d_{j}^{1, \ldots, 1}$ is equal to $(-1)^{n-j} S(n, j)$. If $j_{1}=\cdots=$ $j_{n}=1$, then, by (13), we have

$$
(k+1)^{n}=\sum_{j=1}^{n} d_{j}^{1, \ldots, 1}(k+1)_{j} .
$$

By Lemma 5, we have

$$
\begin{aligned}
(-x)^{n} & =\sum_{j=1}^{n} S(n, j)(-x-j+1)_{j} \\
& =\sum_{j=1}^{n} S(n, j)(-1)^{j}\left(x_{j}\right),
\end{aligned}
$$

since $(-x-j+1)_{j}=(-x-j+1)(-x-j+2) \cdots(-x)=(-1)^{j}(x)_{j}$. Thus we obtain

$$
x^{n}=\sum_{j=1}^{n}(-1)^{n-j} S(n, j)(x)_{j} .
$$

If we compare (20) and (22), then $d_{j}^{1, \ldots, 1}=(-1)^{n-j} S(n, j)$ for all $1 \leq j \leq n$. Then from (19), it completes the proof of Theorem 1 .

Then the polynomial $G_{n}(\tau)$ can be written as

$$
\begin{aligned}
& G_{n}(\tau) \\
& =\sum_{j=1}^{n}(-1)^{n-j} S(n, j)(j+1) ! \sum_{k=0}^{j}\left(\begin{array}{l}
j \\
k
\end{array}\right)\left(\frac{1}{2}\right)^{j-k} \tau^{k} \\
& =\sum_{k=0}^{n}\left\{\sum_{j=k}^{n}(-1)^{n-j} S(n, j)(j+1) !\left(\begin{array}{l}
j \\
k
\end{array}\right)\left(\frac{1}{2}\right)^{j-k}\right\} \tau^{k} \\
& =\sum_{k=0}^{n} a(n, n-k) \tau^{k},
\end{aligned}
$$

where

$$
\begin{aligned}
& a(n, \ell) \\
& \quad:=\sum_{j=n-\ell}^{n}(-1)^{n-j} S(n, j)(j+1) !\left(\begin{array}{c}
j \\
n-\ell
\end{array}\right)\left(\frac{1}{2}\right)^{j-n+\ell} .
\end{aligned}
$$

Note that

$$
\begin{aligned}
\left(\begin{array}{c}
j \\
n-\ell
\end{array}\right) & =\frac{j(j-1) \cdots(j-n+\ell+1)}{(n-\ell) !} \\
& =\frac{j !}{(n-\ell) !(j-n+\ell) !} \\
& =\frac{(n-\ell+1)(n-\ell+2) \cdots j}{(j-n+\ell) !} \\
& =(-1)^{j-n+\ell} \frac{(\ell-n-1)(\ell-n-2) \cdots(-j)}{(j-n+\ell) !} \\
& =(-1)^{j-n+\ell}\left(\begin{array}{c}
\ell-n-1 \\
\ell-n+j
\end{array}\right) .
\end{aligned}
$$

Thus we have

$$
\begin{aligned}
& a(n, \ell) \\
& =(-1)^{\ell} \sum_{j=n-\ell}^{n} S(n, j)(j+1) !\left(\begin{array}{c}
\ell-n-1 \\
\ell-n+j
\end{array}\right)\left(\frac{1}{2}\right)^{j-n+\ell} .
\end{aligned}
$$

Note that $S(n, j)=0$ for $j>n$ and $\left(\begin{array}{c}\ell-n-1 \\ \ell-n+j\end{array}\right)=0$ for $j<n-\ell$; we have

$$
\begin{aligned}
& a(n, \ell) \\
& \quad=(-1)^{\ell} \sum_{j=0}^{\infty} S(n, j) j !(j+1)\left(\begin{array}{l}
\ell-n-1 \\
\ell-n+j
\end{array}\right)\left(\frac{1}{2}\right)^{j-n+\ell} .
\end{aligned}
$$


Lemma 6. For any nonnegative integer t, one has

$$
\sum_{j=0}^{\infty}(j+1)\left(\begin{array}{c}
-t-1 \\
-t+j
\end{array}\right) x^{j}=\frac{d}{d x}\left(\frac{x^{t+1}}{(1+x)^{t+1}}\right) .
$$

Proof. Note that for any nonnegative integer $t$,

$$
\begin{aligned}
\sum_{j=0}^{\infty} & (j+1)\left(\begin{array}{c}
-t-1 \\
-t+j
\end{array}\right) x^{-t+j} \\
& =\sum_{j=0}^{\infty}(j+t+1)\left(\begin{array}{c}
-t-1 \\
j
\end{array}\right) x^{j} .
\end{aligned}
$$

Note that

$$
\begin{aligned}
& \frac{d}{d x}\left(\sum_{j=0}^{\infty}\left(\begin{array}{c}
-t-1 \\
j
\end{array}\right) x^{j+t+1}\right) \\
& =\sum_{j=0}^{\infty}\left(\begin{array}{c}
-t-1 \\
j
\end{array}\right)(j+t+1) x^{j+t} \\
& =\sum_{j=0}^{\infty}(j+t+1)\left(\begin{array}{c}
-t-1 \\
j
\end{array}\right) x^{j+t} .
\end{aligned}
$$

Thus

$$
\sum_{j=0}^{\infty}(j+t+1)\left(\begin{array}{c}
-t-1 \\
j
\end{array}\right) x^{j}=\frac{1}{x^{t}} \frac{d}{d x}\left(\frac{x^{t+1}}{(1+x)^{t+1}}\right),
$$

which completes the proof.

By Lemma 6, we have

$$
\begin{aligned}
\sum_{j=0}^{\infty} & (j+1)\left(\begin{array}{c}
\ell-n-1 \\
\ell-n+j
\end{array}\right)\left(\frac{1}{2}\right)^{\ell-n+j} u^{j} \\
& =\left(\frac{1}{2}\right)^{\ell-n} \sum_{j=0}^{\infty}(j+1)\left(\begin{array}{c}
\ell-n-1 \\
\ell-n+j
\end{array}\right)\left(\frac{u}{2}\right)^{j} \\
& =\left.\left(\frac{1}{2}\right)^{\ell-n} \frac{d}{d x}\left(\frac{x^{n-\ell+1}}{(1+x)^{n-\ell+1}}\right)\right|_{x=u / 2} .
\end{aligned}
$$

Definition 7. Define

$$
H_{n, \ell}(u):=\left.\left(\frac{1}{2}\right)^{\ell-n} \frac{d}{d x}\left(\frac{x^{n-\ell+1}}{(1+x)^{n-\ell+1}}\right)\right|_{x=u / 2} .
$$

Let $\left[t^{n}\right]$ be the operator which gives the $n$th coefficient in the series expansion of a generating function. It is wellknown that the exponential generating function of $S(n, j)$ is the formal power series

$$
\frac{\left(e^{t}-1\right)^{j}}{j !}=\sum_{n=0}^{\infty} S(n, j) \frac{t^{n}}{n !}
$$

Using the above generating function, we prove the following.
Theorem 8. Let $G_{n}(\tau)$ be the polynomial defined as in (8) with

$$
G_{n}(\tau)=\sum_{k=0}^{n} a_{n}(n-k) \tau^{k}
$$

Then the coefficient $a(n, \ell)$ is written as

$$
a(n, \ell)=(-1)^{\ell}\left[\frac{t^{n}}{n !}\right] H_{n, \ell}\left(e^{t}-1\right),
$$

where $0 \leq \ell \leq n$.

Proof. By (27) and (32), the coefficient $a(n, \ell)$ of $G_{n}(\tau)$ can be expressed as

$$
a(n, \ell)=(-1)^{\ell} \sum_{j=0}^{\infty} S(n, j) j !\left[u^{j}\right] H_{n, \ell}(u)
$$

Note that, by (34), we have

$$
\begin{aligned}
& (-1)^{\ell} \sum_{j=0}^{\infty}\left(e^{t}-1\right)^{j}\left[u^{j}\right] H_{n, \ell}(u) \\
& =(-1)^{\ell} \sum_{j=0}^{\infty} \sum_{n=0}^{\infty} S(n, j) j !\left[u^{j}\right] H_{n, \ell}(u) \frac{t^{n}}{n !} \\
& =\sum_{n=0}^{\infty}\left((-1)^{\ell} \sum_{j=0}^{\infty} S(n, j) j !\left[u^{j}\right] H_{n, \ell}(u)\right) \frac{t^{n}}{n !} \\
& =\sum_{n=0}^{\infty} a(n, \ell) \frac{t^{n}}{n !},
\end{aligned}
$$

which follows that

$$
\begin{aligned}
a(n, \ell) & =(-1)^{\ell}\left[\frac{t^{n}}{n !}\right] \sum_{j=0}^{\infty}\left(e^{t}-1\right)^{j}\left[u^{j}\right] H_{n, \ell}(u) \\
& =(-1)^{\ell}\left[\frac{t^{n}}{n !}\right] H_{n, \ell}\left(e^{t}-1\right) .
\end{aligned}
$$

It completes the proof.

\section{Lu Qi-Keng Domains}

In this section we investigate the explicit form of $a(n, \ell)$ and prove that the Bergman kernel for $D_{n}$ has zeros for any positive integer $n$.

Note that if $(\zeta, z),(\eta, w) \in D_{n}$, then

$$
\begin{aligned}
& \left|\frac{\langle\zeta, \eta\rangle}{\prod_{l=1}^{n}\left(1-z_{l} \bar{w}_{l}\right)}\right| \leq \frac{|\zeta||\eta|}{\prod_{l=1}^{n} \sqrt{\left(1-\left|z_{l}\right|^{2}\right)\left(1-\left|w_{l}\right|^{2}\right)}} \\
& \quad=\sqrt{\frac{|\zeta|^{2}}{\prod_{l=1}^{n}\left(1-\left|z_{l}\right|^{2}\right)} \frac{|\eta|^{2}}{\prod_{l=1}^{n}\left(1-\left|w_{l}\right|^{2}\right)}}<1 .
\end{aligned}
$$

Thus by Theorem 1 and (8), we obtain the following. 
Lemma 9. The zero set

$$
\left\{((\zeta, z),(\eta, w)) \in D_{n} \times D_{n}: K_{n}((\zeta, z),(\eta, w))=0\right\}
$$

is equal to the zero set

$$
\left\{((\zeta, z),(\eta, w)) \in D_{n} \times D_{n}: \widetilde{F}_{n}(t)=0,|t|<1\right\},
$$

where $t:=\langle\zeta, \eta\rangle / \prod_{l=1}^{n}\left(1-z_{l} \bar{w}_{l}\right)$.

Since the holomorphic function $t \mapsto 1 /(1-t)-1 / 2$ maps the unit disk onto the right half plane, we obtain the following consequence of Theorem 1 and Lemma 9.

Lemma 10. For any positive integers $n$, the domain $D_{n}$ is a $L u$ Qi-Keng domain if and only if all zeros of the polynomial $G_{n}(\tau)$ lie in the closed left half plane $\{z \in \mathbb{C}: \operatorname{Re} z \leq 0\}$.

The Routh-Hurwitz criterion is the most efficient method for determining whether the polynomial $G_{n}(\tau)$ has zeros in the open left half plane. Let

$$
f(\tau)=a_{0} \tau^{n}+a_{1} \tau^{-1}+\cdots+a_{n-1} \tau+a_{n},
$$

with real coefficients and $a_{0}>0$, and define $\Delta_{j}^{n}$ for $j=1, \ldots, n$ by

$$
\Delta_{j}^{n}:=\left|\begin{array}{ccccc}
a_{1} & a_{3} & a_{5} & \cdots & a_{2 j-1} \\
a_{0} & a_{2} & a_{4} & \cdots & a_{2 j-2} \\
0 & a_{1} & a_{3} & \cdots & a_{2 j-3} \\
\vdots & \vdots & \vdots & \ddots & \vdots \\
0 & 0 & 0 & \cdots & a_{j}
\end{array}\right|,
$$

where $a_{j}=0$ if $j<0$ or $j>n$.

Lemma 11 (Routh-Hurwitz/Liénard-Chipart [15]). All zeros of given polynomial $f(\tau)$ lie in the open left half plane $\{\tau \in \mathbb{C}$ : $\operatorname{Re} \tau<0\}$ if and only if

$$
\Delta_{1}^{n}>0, \ldots, \Delta_{n}^{n}>0 .
$$

This condition is also equivalent to any one of the following four forms:

(i) $a_{n}>0, a_{n-2}>0, a_{n-4}>0, \ldots ; \Delta_{1}^{n}>0, \Delta_{3}^{n}>0, \ldots$,

(ii) $a_{n}>0, a_{n-2}>0, a_{n-4}>0, \ldots ; \Delta_{2}^{n}>0, \Delta_{4}^{n}>0, \ldots$,

(iii) $a_{n}>0 ; a_{n-1}>0, a_{n-3}>0, \ldots ; \Delta_{1}^{n}>0, \Delta_{3}^{n}>0, \ldots$,

(iv) $a_{n}>0 ; a_{n-1}>0, a_{n-3}>0, \ldots ; \Delta_{2}^{n}>0, \Delta_{4}^{n}>0, \ldots$

Proposition 12. Let $\left[t^{n}\right]$ be the operator which gives the $n t h$ coefficient in the series expansion of a generating function

$$
a(n, \ell)=(-1)^{\ell}(n-\ell+1) 2^{n-\ell+2}\left[\frac{t^{n}}{n !}\right] \frac{\left(e^{t}-1\right)^{n-\ell}}{\left(e^{t}+1\right)^{n-\ell+2}} .
$$

Proof. Note that

$$
\begin{aligned}
H_{n, \ell}(u) & =\left.\left(\frac{1}{2}\right)^{\ell-n} \frac{d}{d x}\left(\frac{x^{n-\ell+1}}{(1+x)^{n-\ell+1}}\right)\right|_{x=u / 2} \\
& =\left.\left(\frac{1}{2}\right)^{\ell-n}(n-\ell+1) \frac{x^{n-\ell}}{(1+x)^{n-\ell+2}}\right|_{x=u / 2} \\
& =(n-\ell+1) 2^{n-\ell+2} \frac{u^{n-\ell}}{(u+2)^{n-\ell+2}} .
\end{aligned}
$$

By Theorem 8,

$$
a(n, \ell)=(-1)^{\ell}(n-\ell+1) 2^{n-\ell+2}\left[\frac{t^{n}}{n !}\right] \frac{\left(e^{t}-1\right)^{n-\ell}}{\left(e^{t}+1\right)^{n-\ell+2}} .
$$

By Proposition 12, we have

$$
\begin{gathered}
a(n, n)=4(-1)^{n}\left[\frac{t^{n}}{n !}\right] \frac{1}{\left(e^{t}+1\right)^{2}}, \\
a(n, n-1)=16(-1)^{n-1}\left[\frac{t^{n}}{n !}\right] \frac{e^{t}-1}{\left(e^{t}+1\right)^{3}}, \\
a(n, n-2)=48(-1)^{n-2}\left[\frac{t^{n}}{n !}\right] \frac{\left(e^{t}-1\right)^{2}}{\left(e^{t}+1\right)^{4}} .
\end{gathered}
$$

Theorem 13. For each $n \geq 3$, at least one of $a(n, n), a(n, n-1)$, or $a(n, n-2)$ is negative. More precisely, one has

(i) $a(n, n)<0$ if $n=3,4,7,8,11,12,15,16, \ldots$,

(ii) $a(n, n-1)<0$ if $n=5,9,13, \ldots$,

(iii) $a(n, n-2)<0$ if $n=6,10,14, \ldots$

Proof. We will prove it in Section 4.

Remark 14. (i) Note that

$$
G_{2}(\tau)=\frac{1}{2}+6 \tau+4 \tau^{2}
$$

has two negative real zeros. Thus, $D_{2}$ is a Lu Qi-Keng domain.

(ii) In fact, we see that

$$
G_{3}(\tau)=-\frac{1}{2}+2 \tau+18 \tau^{2}+24 \tau^{3}
$$

has one positive real zero $-1 / 8+\sqrt{33} / 24>0$. Thus, we conclude that the Bergman kernel for $D_{3}$ has zeros, so $D_{3}$ is not a Lu Qi-Keng domain.

(iii) By using a computer program (Maple or Mathematica), we computed the explicit values of $a(n, n), a(n, n-1)$, and $a(n, n-2)$ for $2 \leq n \leq 14$. One can check Theorem 13 holds for $3 \leq n \leq 14$ in Tables 1 and 2 .

Now we can prove the main theorem of this paper using Theorem 13 . 
TABLE $1: 2 \leq n \leq 8$.

\begin{tabular}{lccccccc}
\hline & $G_{2}$ & $G_{3}$ & $G_{4}$ & $G_{5}$ & $G_{6}$ & $G_{7}$ & $G_{8}$ \\
\hline$a(n, n)$ & $1 / 2$ & $-\mathbf{1} / \mathbf{2}$ & $-\mathbf{1}$ & 1 & $17 / 4$ & $-\mathbf{1 7 / 4}$ & -31 \\
$a(n, n-1)$ & 6 & 2 & -8 & $-\mathbf{1 3}$ & 34 & 107 & $\mathbf{- 2 4 8}$ \\
$a(n, n-2)$ & 4 & 18 & 6 & -90 & $-\mathbf{1 2 9}$ & 693 & 1896 \\
\hline
\end{tabular}

TABLE $2: 9 \leq n \leq 14$.

\begin{tabular}{lccccc}
\hline$G_{9}$ & $G_{10}$ & $G_{11}$ & $G_{12}$ & $G_{13}$ & $G_{14}$ \\
\hline 131 & $691 / 2$ & $-\mathbf{6 9 1 / 2}$ & $-\mathbf{5 4 6 1}$ & 5461 & $929569 / 8$ \\
$\mathbf{- 1 2 5 8}$ & 2764 & 20462 & -43688 & $\mathbf{- 8 8 5 8 8 1 / 2}$ & 929569 \\
$\mathbf{- 7 9 2 0}$ & $\mathbf{- 3 4 0 1 4}$ & 126918 & 776661 & -2723175 & $\mathbf{- 4 4 7 2 9 6 7 3 / 2}$ \\
\hline
\end{tabular}

Theorem 2 (again). The Bergman kernel for

$$
\begin{aligned}
D_{n} & =\left\{\left(\zeta, z_{1}, z_{2}, \ldots, z_{n}\right) \in \mathbb{C}^{n+1}:\|\zeta\|^{2}\right. \\
& \left.<\prod_{j=1}^{n}\left(1-\left|z_{j}\right|^{2}\right)\right\}
\end{aligned}
$$

is zero-free if and only if $n \leq 2$.

Proof. (i) If $n=4 k-1$ or $n=4 k$ for $k \in \mathbb{N}$, then $a(n, n)<0$ by Theorem 13 . So, the polynomial $G_{n}(\tau)$ does not satisfy any condition in Lemma 11. It follows that $D_{n}$ is not a Lu Qi-Keng domain.

(ii) If $n=4 k+1$ for $k \in \mathbb{N}$, then $a(n, n-1)<0$ by Theorem 13 . So, the polynomial $G_{n}(\tau)$ does not satisfy conditions (iii) and (iv) in Lemma 11. It follows that $D_{n}$ is not a Lu Qi-Keng domain.

(iii) If $n=4 k+1$ for $k \in \mathbb{N}$, then $a(n, n-2)<0$ by Theorem 13 . So, the polynomial $G_{n}(\tau)$ does not satisfy conditions (i) and (ii) in Lemma 11. It follows that $D_{n}$ is not a Lu Qi-Keng domain.

By (i), (ii), and (iii), $G_{n}(\tau)$ does not satisfy any condition of Routh-Hurwitz theorem, so the Bergman kernel for $D_{n}$ has zeros for all $n \geq 3$.

\section{Proof of Theorem 13}

In this section, we investigate the properties of $a(n, n), a(n, n-$ $1)$, and $a(n, n-2)$ and prove Theorem 13 . For convenience, we denote the functions $A, B, C, D$ by

$$
\begin{aligned}
& A(t):=\frac{1}{e^{t}+1}=\sum_{k=0}^{\infty} a_{k} t^{k}, \\
& B(t):=\frac{1}{\left(e^{t}+1\right)^{2}}=\sum_{k=0}^{\infty} b_{k} t^{k}, \\
& C(t):=\frac{1}{\left(e^{t}+1\right)^{3}}=\sum_{k=0}^{\infty} c_{k} t^{k}, \\
& D(t):=\frac{1}{\left(e^{t}+1\right)^{4}}=\sum_{k=0}^{\infty} d_{k} t^{k} .
\end{aligned}
$$

It is interesting that the numbers $a_{k}$ 's are related to the following Bernoulli numbers. Bernoulli [16] introduced Bernoulli numbers $B_{2 n}$ for $n \geq 1$ satisfying the identity

$$
\frac{t}{e^{t}-1}=1-\frac{t}{2}+\sum_{n=1}^{\infty}(-1)^{n+1} B_{2 n} \frac{t^{2 n}}{(2 n) !}
$$

where $B_{2}=1 / 6, B_{4}=1 / 30$, and $B_{6}=1 / 42, \ldots$ The Genocch $i$ numbers $G_{2 n}$ for $n \geq 1$ are defined [17] by

$$
G_{2 n}:=2\left(2^{2 n}-1\right) B_{2 n}
$$

Lemma 15 (see [18]). Let $n$ be any positive integer. Then

(i) $B_{2 n}$ 's are positive for all positive integers $n$;

(ii) $2 t /\left(e^{t}+1\right)=t+\sum_{n=1}^{\infty}(-1)^{n} G_{2 n}\left(t^{2 n} /(2 n) !\right)$.

Lemma 16. For any $k \in \mathbb{N}$, one has

$$
\begin{aligned}
a_{2 k} & =0, \\
a_{4 k-1} & >0, \\
a_{4 k-3} & <0 .
\end{aligned}
$$

Moreover $a_{0}=1 / 2$.

Proof. By Lemma 15(ii), we have

$$
\frac{1}{e^{t}+1}=\frac{1}{2}+\sum_{n=1}^{\infty} \frac{(-1)^{n} G_{2 n}}{2 \cdot(2 n) !} t^{2 n-1} \text {. }
$$

It follows that

$$
\begin{aligned}
a_{0} & =\frac{1}{2}, \\
a_{2 k} & =0 \quad(k \geq 1), \\
a_{2 n-1} & =(-1)^{n} \frac{G_{2 n}}{2 \cdot(2 n) !} .
\end{aligned}
$$

Note that $G_{2 k}>0$ for all $k \geq 1$ by Lemma 15(i). Thus $a_{4 k-1}>0$ and $a_{4 k-3}<0$ for all $k \geq 1$.

Lemma 17. For any $k \in \mathbb{N}$, one has
(i) $b_{2 k+1}=a_{2 k+1}$,
(ii) $b_{k}=(k+1) a_{k+1}+a_{k}$,
(iii) $2 c_{2 k}=3 b_{2 k}$,
(iv) $2 c_{k}=(k+1) b_{k+1}+2 b_{k}$,
(v) $2 d_{2 k+1}=4 a_{2 k+1}-6 b_{2 k+1}+4 c_{2 k+1}$,
(vi) $3 d_{k}=(k+1) c_{k+1}+3 c_{k}$.

Proof. From the identity

$$
B(-t)=\frac{e^{2 t}}{\left(e^{t}+1\right)^{2}}=\frac{\left(e^{t}+1\right)^{2}-2\left(e^{t}+1\right)+1}{\left(e^{t}+1\right)^{2}},
$$


we obtain

$$
B(t)-B(-t)=2 A(t)-1 \text {. }
$$

It follows that

$$
2 \sum_{k=0}^{\infty} b_{2 k+1} t^{2 k+1}=2 \sum_{k=0}^{\infty} a_{k} t^{k}+1
$$

Thus we obtain (i). From the identity

$$
A^{\prime}(t)=\frac{-e^{t}}{\left(e^{t}+1\right)^{2}}=\frac{-\left(e^{t}+1\right)+1}{\left(e^{t}+1\right)^{2}}
$$

we obtain

$$
A(t)+A^{\prime}(t)=B(t)
$$

It follows that

$$
\sum_{k=0}^{\infty} a_{k} t^{k}+\sum_{k=1}^{\infty} k a_{k} t^{k-1}=\sum_{k=0}^{\infty} b_{k} t^{k}
$$

Thus we obtain (ii). From the identity

$$
C(t)+C(-t)=1-3 A(t)+3 B(t),
$$

we have $2 c_{2 k}=-3 a_{2 k}+3 b_{2 k}$. Since $a_{2 k}=0$ for $k \geq 1$ in Lemma 16, we obtain (iii). From the identity

$$
B^{\prime}(t)+2 B(t)=2 C(t),
$$

we obtain (iv). Similarly as the previous proofs, we can easily see that $(v)$ comes from the identity

$$
D(t)-D(-t)=-1+4 A(t)-6 B(t)+4 C(t),
$$

and (vi) comes from the identity

$$
C^{\prime}(t)+3 C(t)=3 D(t) .
$$

Proposition 18. For any $k \in \mathbb{N}$, it holds that

(i) $a_{2 k}=0, a_{4 k-1}>0, a_{4 k+1}<0$,

(ii) $b_{4 k}<0, b_{4 k+1}<0, b_{4 k+2}>0, b_{4 k+3}>0$,

(iii) $c_{4 k+1}>0, c_{4 k+2}>0$,

(iv) $d_{4 k+2}<0$ for $k \geq 2$.

Proof. (ii) Note that

$$
\begin{aligned}
b_{4 k} & =(4 k+1) a_{4 k+1}<0, \\
b_{4 k+1} & =a_{4 k+1}<0, \\
b_{4 k+2} & =(4 k+3) a_{4 k+3}>0, \\
b_{4 k+3} & =a_{4 k+3}>0 .
\end{aligned}
$$

(iii) Note that $c_{4 k+2}=(3 / 2) b_{4 k+2}>0$. Since $C(t)=A(t)$. $B(t)$, we have

$$
\begin{aligned}
c_{4 k+1} & =\sum_{l=0}^{4 k+1} a_{l} b_{4 k+1-l} \\
& =a_{0} b_{4 k+1}+a_{1} b_{4 k}+a_{4 k+1} b_{0}+\sum_{l=1}^{2 k-1} a_{2 l+1} b_{4 k-2 l} \\
& >a_{0} b_{4 k+1}+a_{1} b_{4 k}+a_{4 k+1} b_{0} \\
& =\frac{1}{2} b_{4 k+1}-\frac{1}{4} b_{4 k}+\frac{1}{4} a_{4 k+1} \\
& =\frac{1}{2} a_{4 k+1}-\frac{1}{4}(4 k+1) a_{4 k+1}+\frac{1}{4} a_{4 k+1} \\
& =\frac{1}{4}(2-4 k) a_{4 k+1}>0 .
\end{aligned}
$$

Here by Proposition 18(i) and (ii),

$$
\sum_{l=1}^{2 k-1} a_{2 l+1} b_{4 k-2 l}=\sum_{l=1}^{k-1} a_{4 l+1} b_{4 k-4 l}+\sum_{l=1}^{k} a_{2 l-1} b_{4 k-4 l+2}>0 .
$$
$k \geq 2$

(iv) Similarly as the proof of (iii), we obtain $d_{4 k+2}<0$ for Now we prove Theorem 13 using the above proposition.

Theorem 13 (again). For any positive integers $k$, one has

(i) $a(n, n)<0$ if $n=4 k-1$ or $n=4 k$,

(ii) $a(n, n-1)<0$ if $n=4 k+1$,

(iii) $a(n, n-2)<0$ if $n=4 k+2$.

Proof. (i) Note that

$$
a(n, n)=4(-1)^{n}\left[\frac{t^{n}}{n !}\right] \frac{1}{\left(e^{t}+1\right)^{2}}=4(-1)^{n} b_{n} .
$$

By Proposition 18(ii), it follows that

$$
\begin{gathered}
a(4 k-1,4 k-1)=-4 b_{4 k-1}<0, \\
a(4 k, 4 k)=4 b_{4 k}<0 .
\end{gathered}
$$

(ii) Note that

$$
\begin{aligned}
a(n, n-1) & =16(-1)^{n-1}\left[\frac{t^{n}}{n !}\right] \frac{e^{t}-1}{\left(e^{t}+1\right)^{3}} \\
& =16(-1)^{n-1}\left(b_{n}-2 c_{n}\right)
\end{aligned}
$$

since

$$
\frac{e^{t}-1}{\left(e^{t}+1\right)^{3}}=\frac{\left(e^{t}+1\right)-2}{\left(e^{t}+1\right)^{3}}=\frac{1}{\left(e^{t}+1\right)^{2}}-\frac{2}{\left(e^{t}+1\right)^{3}} .
$$

By Proposition 18(ii) and (iii), it follows that

$$
a(4 k+1,4 k)=16\left(b_{4 k+1}-2 c_{4 k+1}\right)<0 .
$$


(iii) Note that

$$
\begin{aligned}
a(n, n-2) & =48(-1)^{n-2}\left[\frac{t^{n}}{n !}\right] \frac{\left(e^{t}-1\right)^{2}}{\left(e^{t}+1\right)^{4}} \\
& =48(-1)^{n-2}\left(b_{n}-4 c_{n}+4 d_{n}\right),
\end{aligned}
$$

since

$$
\begin{aligned}
\frac{\left(e^{t}-1\right)^{2}}{\left(e^{t}+1\right)^{4}} & =\frac{\left(e^{t}+1-2\right)^{2}}{\left(e^{t}+1\right)^{4}} \\
& =\frac{1}{\left(e^{t}+1\right)^{2}}-\frac{4}{\left(e^{t}+1\right)^{3}}+\frac{4}{\left(e^{t}+1\right)^{4}} .
\end{aligned}
$$

By Lemma 17(iii), we have

$$
\begin{aligned}
a(4 k+2,4 k) & =48\left(b_{4 k+2}-4 c_{4 k+2}+4 d_{4 k+2}\right) \\
& =48\left(-5 b_{4 k+2}+4 d_{4 k+2}\right)<0
\end{aligned}
$$

for $k \geq 2$.

Moreover $a(6,4)=48\left(-5 b_{6}+4 d_{6}\right)=48 \cdot(-43 / 11520)<$ 0 .

\section{Appendix}

We add the explicit forms of Taylor expansions for some functions which have been discussed in Section 4 .

$$
\begin{aligned}
& \frac{1}{e^{x}+1}=\frac{1}{2}-\frac{x}{4}+\frac{x^{3}}{48}-\frac{x^{5}}{480}+\frac{17 x^{7}}{80640} \\
& -\frac{31 x^{9}}{1451520}+\frac{691 x^{11}}{319334400}-\cdots \text {, } \\
& \frac{1}{\left(e^{x}+1\right)^{2}}=\frac{1}{4}-\frac{x}{4}+\frac{x^{2}}{16}+\frac{x^{3}}{48}-\frac{x^{4}}{96}-\frac{x^{5}}{480}+\frac{17 x^{6}}{11520} \\
& +\frac{17 x^{7}}{80640}-\frac{31 x^{8}}{161280}-\frac{31 x^{9}}{1451520} \\
& +\frac{691 x^{10}}{29030400}+\frac{691 x^{11}}{319334400} \\
& -\frac{5461 x^{12}}{1916006400}-\cdots \text {, } \\
& \frac{1}{\left(e^{x}+1\right)^{3}}=\frac{1}{8}-\frac{3 x}{16}+\frac{3 x^{2}}{32}-\frac{x^{4}}{64}+\frac{3 x^{5}}{1280}+\frac{17 x^{6}}{7680} \\
& -\frac{x^{7}}{1792}-\frac{31 x^{8}}{107520}+\frac{x^{9}}{10240} \\
& +\frac{691 x^{10}}{19353600}-\frac{53 x^{11}}{3548160} \\
& -\frac{5461 x^{12}}{1277337600}+\frac{8507 x^{13}}{4025548800} \\
& +\frac{929569 x^{14}}{1859803545600}-\cdots \text {, }
\end{aligned}
$$

$$
\begin{aligned}
\frac{1}{\left(e^{x}+1\right)^{4}}= & \frac{1}{16}-\frac{x}{8}+\frac{3 x^{2}}{32}-\frac{x^{3}}{48}-\frac{3 x^{4}}{256}+\frac{13 x^{5}}{1920} \\
& +\frac{7 x^{6}}{7680}-\frac{107 x^{7}}{80640}+\frac{x^{8}}{215040} \\
& +\frac{629 x^{9}}{2903040}-\frac{41 x^{10}}{2150400} \\
& -\frac{10231 x^{11}}{319334400}+\frac{7127 x^{12}}{1459814400} \\
& +\frac{885881 x^{13}}{199264665600}-\frac{1710341 x^{14}}{1859803545600} \\
& -\frac{24688759 x^{15}}{41845579776000}+\cdots, \\
\frac{e^{x}-1}{\left(e^{x}+1\right)^{3}}= & \frac{x}{8}-\frac{x^{2}}{8}+\frac{x^{3}}{48}+\frac{x^{4}}{48}-\frac{13 x^{5}}{1920}-\frac{17 x^{6}}{5760}
\end{aligned}
$$$$
+\frac{107 x^{7}}{80640}+\frac{31 x^{8}}{80640}-\frac{629 x^{9}}{2903040}
$$$$
-\frac{691 x^{10}}{14515200}+\frac{10231 x^{11}}{319334400}
$$$$
+\frac{5461 x^{12}}{958003200}-\frac{885881 x^{13}}{199264665600}
$$$$
-\cdots,
$$

$$
\begin{aligned}
\frac{\left(e^{x}-1\right)^{2}}{\left(e^{x}+1\right)^{4}}= & \frac{x^{2}}{16}-\frac{x^{3}}{16}+\frac{x^{4}}{192}+\frac{x^{5}}{64}-\frac{43 x^{6}}{11520}-\frac{11 x^{7}}{3840} \\
& +\frac{79 x^{8}}{80640}+\frac{11 x^{9}}{24192}-\frac{5669 x^{10}}{29030400} \\
& -\frac{641 x^{11}}{9676800}+\frac{258887 x^{12}}{7664025600} \\
& +\frac{133 x^{13}}{14598144}-\frac{14909891 x^{14}}{2789705318400} \\
& -\cdots
\end{aligned}
$$

\section{Competing Interests}

The author declares that there is no conflict of interests regarding the publication of this paper.

\section{Acknowledgments}

This work was supported by NRF-2015R1D1A1A01060295 from National Research Foundation of Korea.

\section{References}

[1] J. P. D’Angelo, "A note on the Bergman kernel," Duke Mathematical Journal, vol. 45, no. 2, pp. 259-265, 1978. 
[2] J. P. D’Angelo, "An explicit computation of the Bergman kernel function," The Journal of Geometric Analysis, vol. 4, no. 1, pp. 23-34, 1994.

[3] K. Oeljeklaus, P. Pflug, and E. H. Youssfi, "The Bergman kernel of the minimal ball and applications," Annales de l'Institut Fourier, vol. 47, no. 3, pp. 915-928, 1997.

[4] J.-D. Park, "New formulas of the Bergman kernels for complex ellipsoids in $\mathrm{C}_{2}$," Proceedings of the American Mathematical Society, vol. 136, no. 12, pp. 4211-4221, 2008.

[5] J.-D. Park, "Explicit formulas of the Bergman kernel for 3dimensional complex ellipsoids," Journal of Mathematical Analysis and Applications, vol. 400, no. 2, pp. 664-674, 2013.

[6] A. Yamamori, "The Bergman kernel of the Fock-BARgmannHartogs domain and the polylogarithm function," Complex Variables and Elliptic Equations, vol. 58, no. 6, pp. 783-793, 2013.

[7] W. Yin, K. Lu, and G. Roos, "New classes of domains with explicit Bergman kernel," Science in China Series A, vol. 47, no. 3, pp. 352-371, 2004.

[8] H. Ahn and J.-D. Park, "The explicit forms and zeros of the Bergman kernel function for Hartogs type domains," Journal of Functional Analysis, vol. 262, no. 8, pp. 3518-3547, 2012.

[9] H. Ishi, J.-D. Park, and A. Yamamori, "Bergman kernel function for hartogs domains over bounded homogeneous domains," The Journal of Geometric Analysis, 2016.

[10] H. P. Boas, "The Lu Qi-Keng conjecture fails generically," Proceedings of the American Mathematical Society, vol. 124, no. 7, pp. 2021-2027, 1996.

[11] H. P. Boas, S. Fu, and E. J. Straube, "The Bergman kernel function: explicit formulas and zeroes," Proceedings of the American Mathematical Society, vol. 127, no. 3, pp. 805-811, 1999.

[12] N. Nikolov and W. Zwonek, "The Bergman kernel of the symmetrized polydisc in higher dimensions has zeros," Archiv der Mathematik, vol. 87, no. 5, pp. 412-416, 2006.

[13] L. Zhang and W. Yin, "Lu Qi-Keng's problem on some complex ellipsoids," Journal of Mathematical Analysis and Applications, vol. 357, no. 2, pp. 364-370, 2009.

[14] R. Merris, Combinatorics, John Wiley \& Sons, New York, NY, USA, 2nd edition, 2003.

[15] F. R. Gantmacher, The Theory of Matrices, vol. 1, AMS Chelsea Publishing, Providence, RI, USA, 1998, Translated from the Russian by K. A. Hirsch, Reprint of the 1959 Translation.

[16] J. Bernoulli, Ars conjectandi, opus posthumum. Accedit Tractatus de seriebus infinitis, et epistola gallicé scripta de ludo pilae reticularis, Impensis Thurnisiorum, Fratrum, Basel, Switzerland, 1713.

[17] A. Genocchi, "Intorno allespressione generale denumeri Bernulliani," Annali di Scienze Matematiche e Fisiche, vol. 3, pp. 395-405, 1852.

[18] D. Foata, "Eulerian polynomials: from Euler's time to the present," in The legacy of Alladi Ramakrishnan in the Mathematical Sciences, pp. 253-273, Springer, New York, NY, USA, 2010. 


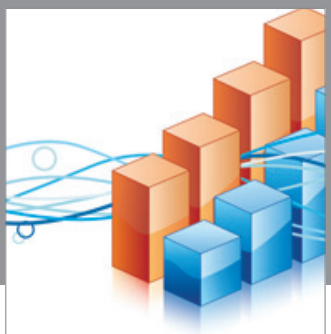

Advances in

Operations Research

vatem alat4

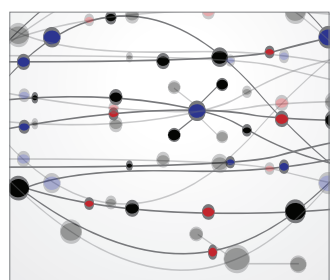

\section{The Scientific} World Journal
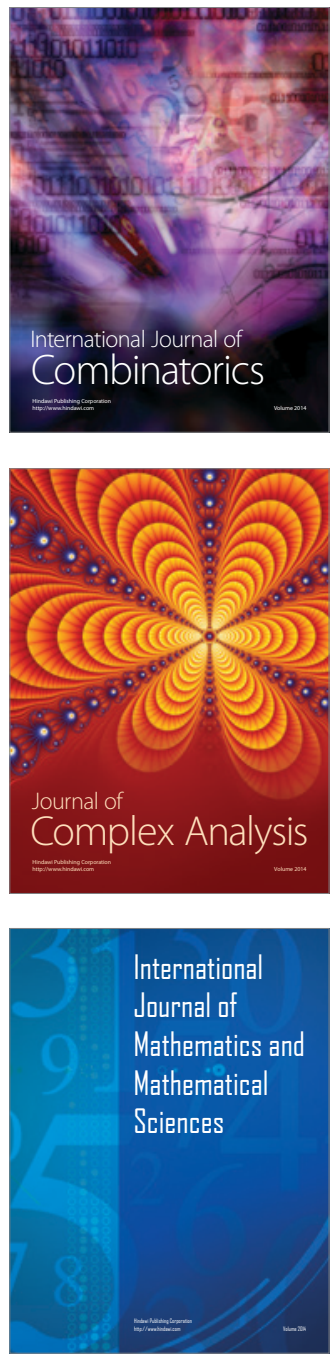
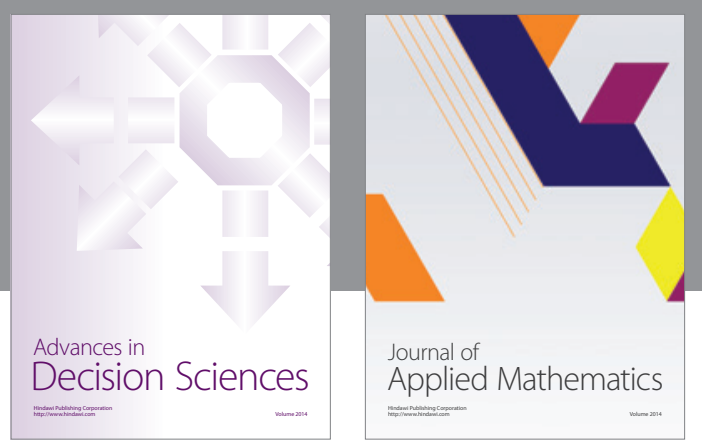

Algebra

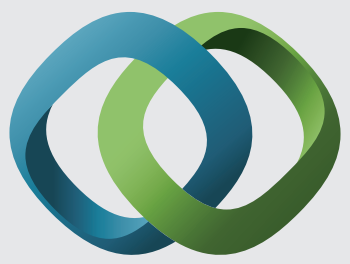

\section{Hindawi}

Submit your manuscripts at

http://www.hindawi.com
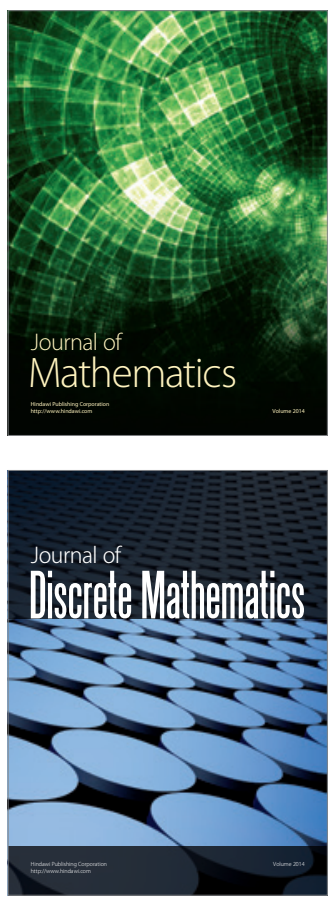

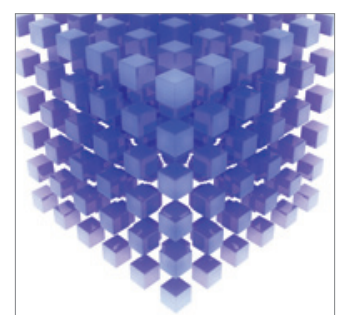

Mathematical Problems in Engineering
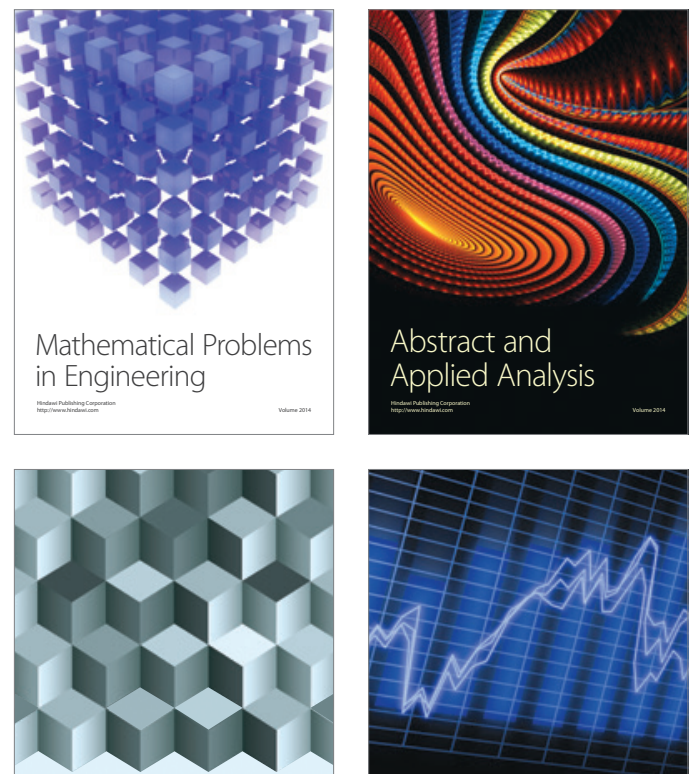

Journal of

Function Spaces

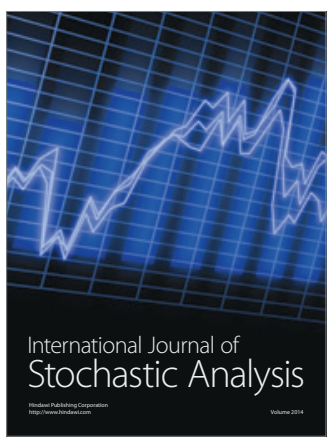

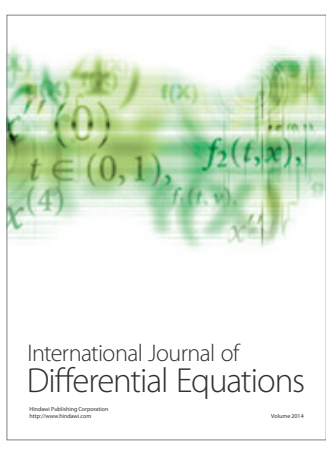
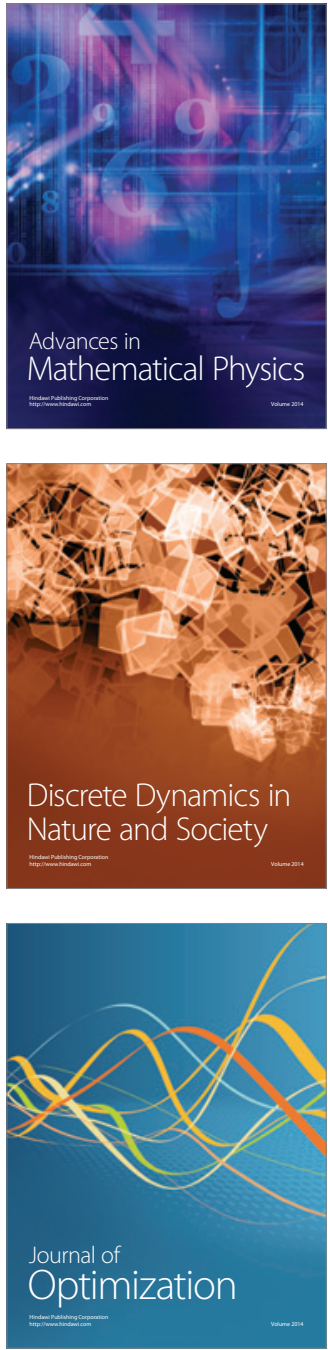